Proyecciones Journal of Mathematics

Vol. 30, No 3, pp. 329-350, December 2011.

Universidad Católica del Norte

Antofagasta - Chile

\title{
Evolution of Weyl's Gauge Invariant Geometry under Ricci Flow
}

\author{
SANDEEP K. BAHUGUNA \\ HNB GARHWAL UNIVERSITY, INDIA \\ and \\ KAILASH C. PETWAL \\ HNB GARHWAL UNIVERSITY, INDIA \\ Received : January 2011. Accepted : September 2011
}

\begin{abstract}
There is a classical fact conjectured by Albert Einstein, that the presence of matter causes the curvature of space-time. However, even a vacant space-time can have a non-zero Weyl's curvature. For instance, such a condition can be found near black holes and in the zones where gravitation waves radiate. Getting inspirations from such a fabulous classical fact, authors have attempted to describe the purely differential geometric behaviour of Weyl's-Gauge invariant conceptions concerning to 4-dimensional structured cosmos. Under the well known Ricci flow (R.F.) techniques, various Weylian configurations have been evolved as heat diffusion equations, which can pave the way for new consequencies in relativity theory and cosmology.
\end{abstract}

M.S.C [2000] : 34B20; 70S15; 58J65

Keywords : Diffusion; Ricci flow (R.F.); Gauge; Cosmos; Weyl; Tensor density; Conformal; rescaling; pseudo vector. 
Nomenclature: $1 . \nabla_{g}^{2}=$ Laplacian operator 2. Semicolon $(;)=$ Covariant differentiation with respect to symmetric affine connection $\Gamma_{j k}^{i} 3$. Comma $()=$, Partial differentiation with respect to the co-ordinates $x^{i}$.

\section{Introduction:}

In the present era of Einsteins theory of relativity, it has been well known that the modern differential geometric tools can easily pave the ways to explain integrated analysis of physical incidents. Different prospects for the elucidation of the fundamental nature and fabric of cosmos in an exactly differential geometric fashion entails that there must be a deep underlying structural basis for extraordinary synchronization that lies in the depth of natures unity. Various Physicists and Mathematicians used to evoke that the cosmos is a self delineating continuum which bond whatsoever seems to be genuinely intrinsic geometric object to physical observable. Recently, various multi-scale differential geometric approaches are being employed up to some great extent to enhance basic outcomes of the Einsteins theory of relativity and it has been noteworthy that the analytic differential geometry alone is able to offer the insightful description of the difficulty and harmony of our structured cosmos that has escort mathematicians and physicists to embark on Geometrization of cosmos and its apparently systematic principles of nature.

The aforementioned prosperity of intrinsic multi scale differential geometric prospects for the geometrization of cosmos theory has produced in the fantastic way of unified field theory. The origin of such unified field theory for structured cosmos can thought to be initiated roughly since 1918, when Prof. Hermann Weyl applied his self-styled purely infinitesimal geometry. This famous Weyls geometry has been immensely used by various researches to geometrize the electromagnetic field of structured cosmos with the hope that it could be incorporated with the already geometrized gravitational field of Einsteins general relativity [5]. In the general relativity, the gravitational field forms the basic geometric structure of a structures cosmos (i.e., usual 4-dimensional continuum), while the electromagnetic field is unconnected to the geometry of such a continuum [7]. Various efforts have been made to establish a new hypothesis of gravitation and electromagnetism with some modified geometry so that there could be room and demand for the prologue of some other tensorial structures besides the well known Riemannian metric tensor. One of the niftiest among these efforts is unquestionably H. Weyls gauge-invariant geometry [7]. Hereafter, we now briefly delineate Weyls gaugeinvariant geometry as such geometry is the main concern of our study.

In the metric manifolds, the space-time interval $d t$ between two infinitesimally near world points with spatial co-ordinates $P\left(x^{i}, t\right)$ and $\left(x^{i}+d x^{i}, t+d t\right)$ is invariant. Thus to each world point, there may concern an invariant "cone" with directions along which $d t$ vanished. Such a cone in the cosmos theory is known as "light cone". Weyl had introduced an idea to amend the geometry of structured cosmos so that the invariance of light cone could be maintained, whereas $d t$ would lose its invariant 
attitude. In addition, to justify the feature of light that "possible directions of light rays are an invariant property of structured cosmos", Weyl also assumed that the zero directions of light rays are entirely distinguishes by the ration between different components of the metric tensor used for structured cosmos and therefore together with co-ordinate transformations of tensorial structures, the existence of "Gauge transformations" would be applicable. The gauge transformation was considered in a fashion, such that it multiplies all components of metric tensor by a factor which is an arbitrary function of spatial co-ordinates. In addition to invariance nature of space-time interval and thus the light cone, the concept of "normalized condition" has been introduced for the arbitrary factor of line element with the pre-assumption that $g_{i j}$ be equal to -1 , i.e.,

$$
\left|g_{i j}\right|=-1 \text {. }
$$

Such normalizing condition would not be invariant if we wish to preserve the tensor transformation character of the metric tensor $g_{i j}$. To avoid this difficulty, we assume that in the present study such metric tensor transforms as the component of a tensor density of weight $\omega=-\frac{1}{2}$ just like below:

$$
\begin{gathered}
\bar{g}_{i j}=g_{\alpha \beta}\left|\frac{\partial x}{\partial \bar{x}}\right|^{-1 / 2} \frac{\partial x^{\alpha}}{\partial \bar{x}^{i}} \frac{\partial x^{\beta}}{\partial \bar{x}^{j}} . \\
\Rightarrow \bar{g}_{i j}=g_{\alpha \beta}\left|\frac{\partial \bar{x}}{\partial x}\right|^{1 / 2} \frac{\partial x^{\alpha}}{\partial \bar{x}^{i}} \frac{\partial x^{\beta}}{\partial \bar{x}^{j}} .
\end{gathered}
$$

The line element $d t=g_{i j} d x^{i} d x^{j}$, thus loses its invariant character. Also, the determinant of the metric tensor can be represented as the multiple product of the metric tensor and Levi-Civita tensor density:

$$
\left|g_{i j}\right| \equiv \frac{1}{24} \delta^{i_{1}, i_{2}, i_{3}, i_{4}} \delta^{j_{1}, j_{2}, j_{3}, j_{4}} g_{i_{1} j_{1}} g_{i_{2} j_{2}} g_{i_{3} j_{3}} g_{i_{4} j_{4}} .
$$

Now, because of the Levi-Civita densities, each having weight +1 , the determinant is therefore a scalar and thus expression (1.4) is an invariant condition. Because of the normalizing condition (1.1), the Riemannian metric tensor involved in this discussion will have only nine independent components.

Let us now discuss some fundamental aspects of Weyl's gauge geometry, which would serve as an especial environment to its evolution by means of Ricci flow.

\subsection{Basic Mathematical Environment of Weyl's Gauge Invariant Geometry:}

In addition to establish evolution equations for the Weyl's gauge invariant geometry under Ricci flow, we introduce some basic tensor calculus involving the notions of affine connection and curvature tensor for the structured cosmos. Since the tensor densities play extensive role in this geometry, we extend the usual concept of covariant differentiation of tensor densities. For this purpose, we employ a set of 
variables with one index $\phi_{\mu}$, which indulges in the usual expression of the covariant differentiation as like below:

$\nabla_{q} u_{i, j, k \cdots}^{l, m, n \cdots}=\frac{\partial}{\partial x^{q}} \nabla_{q} u_{i, j, k \cdots}^{l, m, n \cdots}+u_{i, j, k \cdots}^{e, m, n \cdots} \Gamma_{e q}^{l}+\cdots-u_{e, j, k \cdots}^{l, m, n \cdots} \Gamma_{i q}^{e}-\cdots-w u_{i, j, k \cdots, \phi_{q}}^{l, m, n \cdots}$

where $w$ is the weight of the tensor density $u_{i, j, k \cdots}^{l, m, n}$. To obtain the transformation law of index $\phi_{\mu}$, it is sufficient to consider the covariant derivatives of a scalar density $D$ of weight $w$. These derivatives form a vector density as follows: $\nabla_{q^{*}} D^{*}=\left|\frac{\partial x^{\alpha}}{\partial x^{* \beta}}\right| \frac{\partial x^{l}}{\partial x^{* q}} \nabla_{l} D$.

Therefore, we have

$\nabla_{l}\left(\left|\frac{\partial x^{\alpha}}{\partial x^{* \beta}}\right|^{w} D\right) \frac{\partial x^{l}}{\partial x^{* q}}-w \phi_{q}^{*}\left|\frac{\partial x^{\alpha}}{\partial x^{* \beta}}\right|^{w} D=\left|\frac{\partial x^{\alpha}}{\partial x^{* \beta}}\right|^{w}\left(\nabla_{l} D-w \phi_{l} D\right) \frac{\partial x^{l}}{\partial x^{* q}}$, and therefore we have

$$
w\left[\nabla_{l}\left(\log \left|\frac{\partial x^{\alpha}}{\partial x^{* \beta}}\right|\right) \frac{\partial x^{l}}{\partial x^{* q}}-w \phi_{q}^{*}\right]=-w \phi_{l} \frac{\partial x^{l}}{\partial x^{* q}} D .
$$

This logarithmic derivative of the determinant can be rewritten in the more simplified form:

$$
\nabla_{l}\left(\log \left|\frac{\partial x^{\alpha}}{\partial x^{* \beta}}\right|\right)=\frac{\partial x^{* \beta}}{\partial x^{\alpha}} \frac{\partial x^{* q}}{\partial x^{l}} \frac{\partial^{2} x^{\alpha}}{\partial x^{* \beta} \partial x^{* q}}=-\frac{\partial x^{\alpha}}{\partial x^{* \beta}} \frac{\partial^{2} x^{* \beta}}{\partial x^{\alpha} \partial x^{l}} .
$$

All the above, consequently lead to the following very desirable transformation law of index $\phi_{\mu}$ :

$$
\phi_{q}^{*}=\phi_{l} \frac{\partial x^{l}}{\partial x^{* q}}+\frac{\partial x^{* \beta}}{\partial x^{\alpha}} \frac{\partial^{2} x^{\alpha}}{\partial x^{* \beta} \partial x^{* q}}=\frac{\partial x^{l}}{\partial x^{* q}}\left(\phi_{l}-\frac{\partial x^{\alpha}}{\partial x^{* \beta}} \frac{\partial^{2} x^{* \beta}}{\partial x^{\alpha} \partial x^{l}}\right)
$$

Now, once we have the concept of covariant differentiation in the sense of tensor densities, it is very logical to postulate that covariant derivatives of the Levi-Civita tensor density vanish. Such condition produces a unique relationship between the pseudo vector $\phi_{l}$ and the components of Christoffel's symbol as given below:

$$
\delta^{\rho l_{2} l_{3} l_{4}} \Gamma_{\rho q}^{l_{1}}+\delta^{l_{1} \rho l_{3} l_{4}} \Gamma_{\rho q}^{l_{2}}+\delta^{l_{1} l_{2} \rho l_{4}} \Gamma_{\rho q}^{l_{3}}+\delta^{l_{1} l_{2} l_{3} \rho} \Gamma_{\rho q}^{l_{4}}-\delta^{l_{1} l_{2} l_{3} l_{4}} \phi_{q}=0 .
$$

By straight forward computation, it can be shown that each term on the left hand side of equation (1.9) is skew symmetric with respect to all the four indices $l_{1}, l_{2}, l_{3}$ and $l_{4}$. Also, in this significant equation, the summation over $\rho$ in the first term reduces to the one value $\rho=l_{1}$, likewise second term $\rho=l_{2}$ and so forth. The first four terms in (1.9) are equal to $\delta^{l_{1} l_{2} l_{3} l_{4}} \Gamma_{\rho q}^{\rho}$ and the condition (1.9) then reduces to the form: 


$$
\phi_{\sigma}=\Gamma_{\rho \delta}^{\rho} .
$$

Moreover, the metric tensor in Weyl's geometry plays a role similar to that the metric tensor in Riemannian geometry. we shall therefore assume that its components of covariant differentiation vanish and the Christoffel's symbol are symmetric in their subscripts. Now we have an expression:

$$
g_{\mu \nu ; \rho} \equiv g_{\mu \nu, \rho}-g_{\mu \sigma} \Gamma_{\nu \rho}^{\sigma}-g_{\sigma \nu} \Gamma_{\mu \rho}^{\sigma}+\frac{1}{2} g_{\mu \nu} \phi_{\rho}=0 .
$$

These equations can be solved, if we introduce the "contravariant metric tensor density" $g^{\mu \nu}$ of weight $\left(+\frac{1}{2}\right)$ which has the property $g_{l \mu} g^{\mu \nu}=\delta_{l}^{\nu}$.

Hence the solution of equation (1.11) then takes the form:

$$
\Gamma_{l k}^{\lambda}=\frac{1}{2} g^{\lambda \sigma}\left(g_{l \sigma, k}+g_{k \sigma, l}-g_{l k, \sigma}\right)+\frac{1}{4} g^{\lambda \sigma}\left(g_{l \sigma} \phi_{k}+g_{k \sigma} \phi_{l}-g_{l k} \phi_{\sigma}\right) \equiv\left(\begin{array}{c}
\lambda \\
l k
\end{array}\right) .
$$

If we reform the contracted Christoffel's symbol $\left(\begin{array}{l}l \\ l k\end{array}\right)$, we again have equation (1.10). In Weyl's geometry, the pseudo vector $\phi_{l}$ and the Riemannian metric tensor $g_{\mu \nu}$ are independent of each other, but needed to form the components of Christoffel's symbol $\left(\begin{array}{c}\lambda \\ l k\end{array}\right)$. Also, the curvature tensor $R_{l k \lambda}^{\nu}$ is skew symmetric in its first index pair, has the cyclic symmetry as $R_{l k \lambda}^{\nu}+R_{k \lambda i}^{\nu}+R_{\lambda l k}^{\nu}=0$ and satisfies the well known Bianchi's identity given by

$$
R_{l k \sigma ; \lambda}^{\nu}+R_{k \lambda \sigma ; l}^{\nu}+R_{\lambda l \sigma ; k}^{\nu}=0 .
$$

If we contract this Bianchi's identity with respect to indices $l$ and $\nu$, we have the equation:

$$
R_{\rho k \sigma ; \lambda}^{\rho}+R_{k \lambda \sigma ; \rho}^{\rho}+R_{\lambda \rho \sigma ; k}^{\rho}=R_{\rho k \sigma ; \lambda}^{\rho}+R_{k \lambda \sigma ; \rho}^{\rho}-R_{\rho \lambda \sigma ; k}^{\rho} \equiv 0 .
$$

Let us now raise the index $\sigma$ and contract the equation (1.14) with respect to $k$ and $\sigma$, we obtain an equation of the form:

$$
R_{\rho \sigma ; \lambda}^{\sigma \rho}+R_{\sigma \lambda ; \rho}^{\sigma \rho}-R_{\rho \lambda ; \sigma}^{\sigma \rho} \equiv 0
$$

in which the $R$-quantities are densities.

In Riemannian geometry, due to the skew symmetric property of curvature tensor, the second and third terms of equation (1.15) are equal, while in case of Weyl's geometry the same reason can not be applied. Therefore to obtain symmetric relationship of last index pair in this geometry, one need to form a 
covariant curvature density $R_{l k \lambda \mu}$. Thus in Weyl's geometry the following symbol has been adopted:

$$
(l k, \lambda)=g_{\lambda \sigma}\left(\begin{array}{c}
\sigma \\
l k
\end{array}\right)=\frac{1}{2}\left(g_{l \lambda, k}+g_{k \lambda, l}-g_{l k, \lambda}\right)+\frac{1}{4}\left(g_{l \lambda} \phi_{k}+g_{k \lambda} \phi_{l}-g_{l k} \phi_{\lambda}\right) .
$$

In terms of the symbol $(l k, \lambda)$, the derivative of the metric tensor are

$$
g_{l k, \lambda}=(k \lambda, l)+(l \lambda, k)-\frac{1}{2} g_{l k} \phi_{\lambda} .
$$

Furthermore, in the light of symbol $(l k, \lambda)$, the covariant components of curvature tensor density are written in the form:

$$
\begin{gathered}
R_{l k \lambda \mu}^{*}=(\lambda l, \mu)_{, k}+\frac{1}{2}(\lambda l, \mu) \phi_{k}-(\lambda k, \mu)_{, l}-\frac{1}{2}(\lambda k, \mu) \phi_{l} \\
+g^{\rho \sigma}[(\lambda k, \rho)(\mu l, \sigma)-(\lambda l, \rho)(\mu k, \sigma)] \\
=\bar{R}_{l k \lambda \mu}+\frac{1}{4}\left[g_{l \mu} \phi_{\lambda, k}-g_{k, \mu} \phi_{\lambda, l}+g_{\lambda \mu}\left(\phi_{l, k}-\phi_{k, l}\right)+g_{k \lambda} \phi_{\mu, l}-g_{l \lambda} \phi_{\mu, k}\right] .
\end{gathered}
$$

Here in the right hand side of the expression (1.18), $\bar{R}_{l k \lambda \mu}$ stands for a number of terms which have all the algebraic symmetry properties of the Riemannian curvature tensor, while rest of the terms do not have such properties. If we use an expression $R_{l k \lambda \mu}+R_{l k \mu \lambda}$, which vanishes in Riemannian geometry,, we have from equation (1.18):

$$
R_{l k \lambda \mu}^{*}=-R_{l k \mu \lambda}^{*}+2 g_{\lambda \mu}\left(\phi_{l, k}-\phi_{k, l}\right) .
$$

Even though, $\phi_{l}$ is not a vector, its skew-symmetric derivative is given as;

$$
\phi_{l k}=\phi_{l, k}-\phi_{k, l}
$$

which forms a tensor and can be demonstrated with the help of equation (1.8). In case, if one apply equation(1.19) in the second term of equation (1.15), we obtain here;

$$
R_{\rho \sigma ; \lambda}^{\sigma \rho}-2 R_{\rho \lambda ; \sigma}^{\sigma \rho}+2 g^{\rho \sigma} \phi_{\sigma \lambda ; \rho} \equiv 0 .
$$
that;

However in equation (1.21), one can introduce the symmetric part of $R_{\rho k \lambda}^{\rho}$, so 


$$
R_{k \lambda}=\frac{1}{2}\left(R_{\rho k \lambda}^{\rho}+R_{\rho \lambda k}^{\rho}\right) .
$$

In the light of equation (1.18), we observe that $R_{k \lambda}$ and $R_{\rho k \lambda}^{\rho}$ are connected by the equation

$$
R_{\rho k \lambda}^{\rho}=R_{k \lambda}+\frac{1}{2} \phi_{\lambda k} .
$$

Now, by substituting this expression in equation (1.21), we obtain the contracted Bianchi identities in the following form:

$$
\left(R^{\lambda \sigma}-\frac{1}{2} g^{\lambda \mu} R\right)_{; \sigma}+\frac{1}{2} \phi_{; \sigma}^{\lambda \sigma}=0 .
$$

$\phi^{\lambda \sigma}$ is a tensor density of weight +1 and $\phi_{; \sigma}^{\lambda \sigma}$ is therefore the ordinary divergence of $\phi^{\lambda \sigma}$.

In Weyl's geometry, the structured cosmos is characterized by a symmetric tensor density $g_{\mu \nu}$ and the "pseudo vector" $\phi_{\mu}$. In this geometry it is very natural that the $g_{\mu \nu}$ represent gravitational field and the $\phi_{\mu}$ are the components of the world vector potential. In Weyl's's original formalism, the $\phi_{\mu}$ transform as a vector with respect to co-ordinate transformations, but are changed by a gradient when a gauge transformation is applied. This is the historical reason for calling the addition of a gradient to the electromagnetic world vector potential, a gauge transformation.

\subsection{Weyl's-Gauge Invariant Geometry under Ricci Flow:}

The significance of Riemannian geometry in relativity has been questioned both from the axiomatic and observational point of view. For instance, in [6]; the authors have tried to give an axiomatic foundation for the geometrical nature of space-time by means of ideal operations with elementary clocks and rods. Instead of this, a Weylian structure is more appropriate. As from the observational standpoint, one has to keep concentration upon the measuring procedure of invariance of light cone and for this, besides the metric $g_{i j(x)}$, a pseudo scalar function $\phi(x)$ must be introduces. Then such a notion leads a conformally-Riemannian structure which is more specific rather than Riemannian one. Therefore, in the modern study of relativity, the most promising and credible alternatives to Riemannian structures of structured cosmos are the conformally Riemannian (i.e., Weylian configuration)structures.

These Weylian configurations are most prominent because of the two reasons. First, the Weyl's structures possess an additional degree of freedom to involve the Gauge transformations, which make the study of structured cosmos more interesting for dealing with gauge principle in field theory. Second, there are some effects that take place in Weyl's manifold, that classically draw some purely quantum effects. For instance, in [9], the authors verified that there is some kind of 
correlation between objects in classical gravity on Weyl's manifolds and quantum non-Abelian field theory.

Now, the structured cosmos having Weylian structures as its characteristic tools is defined as an affine manifold specified by a metric tensor $g_{i j}(x)$ and a gauge vector (in our case, a pseudo vector $\phi_{i}(x)$ ), such that [4]:

$$
\Upsilon_{j k}^{i}=\Gamma_{j k}^{i}-\frac{1}{2}\left(\phi_{k}+\delta_{j}^{i}+\phi_{j} \delta_{k}^{i}-g_{j k} \phi^{i}\right),
$$

where $\Upsilon_{j k}^{i}$ is Weyl's affine connection and $\Gamma_{j k}^{i}$ is the well known Christoffel's second kind symbol of the metric given by eq. (1.12). By substituting (1.12) in (1.25), we obtain a Weylian affine structure in terms of metric tensor and pseudo vector as follows:

$$
\begin{aligned}
\Upsilon_{j k}^{i}=\frac{1}{2} g^{i h}\left(g_{j h, k}+\right. & \left.g_{k h, j}-g_{j k, h}\right)+\frac{1}{4} g^{i h}\left(g_{j h} \phi_{k}+g_{k h} \phi_{j}-g_{j k} \phi_{h}\right) \\
& -\frac{1}{2}\left(\phi_{k} \delta_{j}^{i}+\phi_{j} \delta_{k}^{i}-g_{j k} \phi^{i}\right) .
\end{aligned}
$$

Furthermore, is has been already remarked that in addition to manifold motions group of Riemannian structures, Weyl's geometry admits internal (i.e. gauge) transformations of the form:

$g_{i j} \rightarrow \Omega^{2}(x) g_{i j}$.

Thereby, the Weyl's tensor can also be characterized as the part of covariant Riemannian tensor:

$$
R_{i k l m}=\frac{\partial}{\partial x^{l}} \Gamma_{i, k m}-\frac{\partial}{\partial x^{m}} \Gamma_{i k, l}+\Gamma_{k l}^{s} \Gamma_{s, i m}-\Gamma_{k m}^{s} \Gamma_{s, i l},
$$

which is covariant under the conformal transformation or deformation of the metric. Thus, if we take the conformal deformation of the metric $g_{i j}$ as;

$$
\widehat{g}_{i j}=\Omega^{2} g_{i j} .
$$

Then, for an $n=4$ dimensional space-time continuum, the Weyl's tensor is given by;

$$
W_{i j k l}=R_{i j k l}-\frac{\left(R_{i k} g_{j l}-R_{i l} g_{j k}+R_{j l} g_{i k}-R_{j k} g_{i l}\right)}{2}+\frac{R\left(g_{i k} g_{j l}-g_{i l} g_{j k}\right)}{6} .
$$


Under the deformation (1.28), this Weyl's tensor takes the form:

$$
\widehat{W}_{i j k l}=\Omega^{2}(x) W_{i j k l} .
$$
form:

Also, under the conformal deformation, the pseudo-vector $\phi_{i}(x)$ will have the

$$
\widehat{\phi}_{i}(x) \rightarrow \phi_{i}(x)+2 \Omega^{-1} \frac{\partial \Omega}{\partial x^{i}} .
$$

The conformal transformations given by equations (1.28) and (1.31) are also acknowledged as Weyl's rescaling of metric tensor and pseudo-vector respectively.

Now, it is well known that while using Weyl's geometry, the properties of symmetries and skew-symmetries for Weyl's tensor can not take place in the same sense as in case of Riemannian geometries. Therefore, we need to develop a covariant Weyl's tensor density $W_{i j k l}^{*}$ as follows:

For the sake of simplicity, concerning to indicial discipline, we rewrite eq. (1.18) as below:

$R_{i j k l}^{*}=\frac{\partial}{\partial x^{j}} \Gamma_{k i, l}+\frac{1}{2} \Gamma_{k i, l} \phi_{j}-\frac{\partial}{\partial x^{i}} \Gamma_{k j, l}-\frac{1}{2} \Gamma_{k j, l} \phi_{l}+g^{\rho \sigma}\left[\Gamma_{k j, \rho} \Gamma_{l i, \sigma}-\Gamma_{k i, \rho} \Gamma_{l j, \sigma}\right]$,

or,

$R_{i j k l}^{*}=\frac{\partial}{\partial x^{j}} g_{h l} \Gamma_{k i}^{h}+\frac{1}{2} \phi_{j} g_{h l} \Gamma_{k i}^{h}-\frac{\partial}{\partial x^{i}} g_{h l} \Gamma_{k j}^{h}-\frac{1}{2} \phi_{i} g_{h l} \Gamma_{k j}^{h}+g_{h \sigma} \times\left[\Gamma_{k j}^{\sigma} \Gamma_{l i}^{h}-\Gamma_{k i}^{\sigma} \Gamma_{l j}^{h}\right]$.

Further, from eq. (1.32), one can calculate the Ricci tensor density (or contracted curvature density) by taking inner product of $g^{i k}$ with eq. (1.32) throughoutly. The result of this process is as follows:

$$
\begin{gathered}
g^{i k} R_{i j k l}^{*}=g^{i k} \frac{\partial}{\partial x^{j}} \Gamma_{k i, l}+\frac{1}{2} \phi_{j} g^{i k} \Gamma_{k i, l}-g^{i k} \frac{\partial}{\partial x^{i}} \Gamma_{k j, l}-\frac{1}{2} g^{i k} \phi_{i} \Gamma_{k j, l} \\
+g^{i k} g^{\rho \sigma}\left[\Gamma_{k j, \rho} \Gamma_{l i, \sigma}-\Gamma_{k i, \rho} \Gamma_{l j, \sigma}\right] . \\
\Rightarrow R_{j l}^{*}=g^{i k} \frac{\partial}{\partial x^{j}}\left(g_{h l} \Gamma_{k j}^{h}\right)+\frac{1}{2} \phi_{j} g^{i k} g_{h l} \Gamma_{k i}^{h}-g^{i k} \frac{\partial}{\partial x^{i}}\left(g_{h l} \Gamma_{k j}^{h}\right)-\frac{1}{2} g^{i k} \phi_{i} g_{h l} \Gamma_{k j}^{h}
\end{gathered}
$$




$$
\begin{gathered}
+g^{i k}\left[\Gamma_{k j}^{\sigma} g_{h \sigma} \Gamma_{l i}^{h}-\Gamma_{k i}^{\sigma} g_{h \sigma} \Gamma_{l j}^{h}\right] . \\
\Rightarrow R_{j l}^{*}=g^{i k} \frac{\partial}{\partial x^{j}}\left(g_{h l} \Gamma_{k j}^{h}\right)+\frac{1}{2} \phi_{j} g^{i k} g_{h l} \Gamma_{k i}^{h}-g^{i k} \frac{\partial}{\partial x^{i}}\left(g_{h l} \Gamma_{k j}^{h}\right)-\frac{1}{2} g^{i k} \phi_{i} g_{h l} \Gamma_{k j}^{h} \\
+g^{i k} g_{h \sigma}\left[\Gamma_{k j}^{\sigma} \Gamma_{l i}^{h}-\Gamma_{k i}^{\sigma} \Gamma_{l j}^{h}\right] .
\end{gathered}
$$

On the basis of eq. (1.34), we can calculate $R_{j k}^{*}, R_{i l}^{*}$ and $R_{i k}^{*}$ as follows:

$$
\begin{gathered}
R_{j k}^{*}=g^{i l} R_{i j k l}^{*}=g^{i l} \frac{\partial}{\partial x^{j}}\left(g_{h l} \Gamma_{k i}^{h}\right)+\frac{1}{2} \phi_{j} g_{h l} g^{i l} \Gamma_{k i}^{h}-g^{i l} \frac{\partial}{\partial x^{i}}\left(g_{h l} \Gamma_{k j}^{h}\right) \\
-\frac{1}{2} \phi_{i} g_{h l} g^{i l} \Gamma_{k j}^{h}+g^{i l} g_{h \sigma}\left[\Gamma_{k j}^{\sigma} \Gamma_{l i}^{h}-\Gamma_{k i}^{\sigma} \Gamma_{l j}^{h}\right] .
\end{gathered}
$$

Now, applying the process of inner multiplication and contraction for further manipulations, the last expression yields;

$$
\begin{gathered}
R_{j k}^{*}=g^{i j} R_{i j k l}^{*}=g^{i l} \frac{\partial}{\partial x^{j}}\left(g_{h l} \Gamma_{k i}^{h}\right)+\frac{1}{2} \phi_{j} \delta_{h}^{i} \Gamma_{k i}^{h}-g^{i l} \frac{\partial}{\partial x^{i}}\left(g_{h l} \Gamma_{k j}^{h}\right) \\
-\frac{1}{2} \phi_{i} \delta_{h}^{i} \Gamma_{k j}^{h}+g^{i l} g_{h \sigma}\left[\Gamma_{k j}^{\sigma} \Gamma_{l i}^{h}-\Gamma_{k i}^{\sigma} \Gamma_{l j}^{h}\right] .
\end{gathered}
$$

At this stage, using the property $\Gamma_{i j}^{i}=\frac{1}{2} \frac{\partial \log \left(g_{i i}\right)}{\partial x^{k}}$, we finally have;

$$
\begin{aligned}
R_{j k}^{*}=g^{i j} R_{i j k l}^{*} & =g^{i l} \frac{\partial}{\partial x^{j}}\left(g_{h l} \Gamma_{k i}^{h}\right)+\frac{1}{4} \phi_{j} \frac{\partial \log \left(g_{i i}\right)}{\partial x^{k}}-g^{i l} \frac{\partial}{\partial x^{i}}\left(g_{h l} \Gamma_{k j}^{h}\right) \\
& -\frac{1}{2} \phi_{i} \Gamma_{k j}^{i}+g^{i l} g_{h \sigma}\left[\Gamma_{k j}^{\sigma} \Gamma_{l i}^{h}-\Gamma_{k i}^{\sigma} \Gamma_{l j}^{h}\right] .
\end{aligned}
$$

In the similar fashion, we have the component $R_{i l}^{*}$ of the Ricci tensor density as

$$
\begin{gathered}
R_{i l}^{*}=g^{j k} R_{i j k l}^{*}=g^{j k} \frac{\partial}{\partial x^{j}}\left(g_{h l} \Gamma_{k i}^{h}\right)+\frac{1}{2} \phi_{j} g^{j k} g_{h k} \Gamma_{k i}^{h}-g^{j k} \frac{\partial}{\partial x^{i}}\left(g_{h l} \Gamma_{k j}^{h}\right) \\
-\frac{1}{2} \phi_{i} g^{j k} g_{h l} \Gamma_{k j}^{h}+g^{j k} g_{h \sigma}\left[\Gamma_{k j}^{\sigma} \Gamma_{l i}^{h}-\Gamma_{k i}^{\sigma} \Gamma_{l j}^{h}\right] .
\end{gathered}
$$



below:

Eventually, we calculate the component $R_{i k}^{*}$ of the Ricci tensor density as

$$
\begin{gathered}
R_{i k}^{*}=g^{j l} R_{i j k l}^{*}=g^{j l} \frac{\partial}{\partial x^{j}}\left(g_{h l} \Gamma_{k j}^{h}\right)+\frac{1}{2} \phi_{j} g^{j l} g_{h l} \Gamma_{k i}^{h}-g^{j l} \frac{\partial}{\partial x^{i}}\left(g_{h l} \Gamma_{k j}^{h}\right) \\
-\frac{1}{2} \phi_{i} g^{j l} g_{h l} \Gamma_{k j}^{h}+g^{j k} g_{h \sigma}\left[\Gamma_{k j}^{\sigma} \Gamma_{l i}^{h}-\Gamma_{k i}^{\sigma} \Gamma_{l j}^{h}\right] .
\end{gathered}
$$

Again, under the process of inner multiplication and contraction, the last result yields;

$$
\begin{gathered}
R_{i k}^{*}=g^{j l} \frac{\partial}{\partial x^{j}}\left(g_{h l} \Gamma_{k i}^{h}\right)+\frac{1}{2} \phi_{j} \Gamma_{k i}^{j}-g^{j l} \frac{\partial}{\partial x^{i}}\left(g_{h j} \Gamma_{k j}^{h}\right)-\frac{1}{4} \phi_{i} \frac{\partial \log g_{j j}}{\partial x^{k}} \\
+g^{j l} g_{h \sigma}\left[\Gamma_{k j}^{\sigma} \Gamma_{l i}^{h}-\Gamma_{k i}^{\sigma} \Gamma_{l i}^{h}\right] .
\end{gathered}
$$

Afterward, we now calculate the scalar curvature tensor density by taking inner product of $g^{i j}$ with $R_{i j}^{*}$. The result of this process is as follows:

$$
\begin{aligned}
R^{*}=g^{i j} R_{i j} & =g^{i j} g^{k l} \frac{\partial}{\partial x^{j}}\left(g_{h l} \Gamma_{k i}^{h}\right)+\frac{1}{4} \phi^{i} \frac{\partial \log g_{k k}}{\partial x^{i}}-g^{i j} g^{k l} \frac{\partial}{\partial x^{i}}\left(g_{h l} \Gamma_{k j}^{h}\right) \\
& -\frac{1}{4} \phi^{j} \frac{\partial \log g_{k k}}{\partial x^{j}}+g^{i j} g^{k j} g_{h \sigma}\left[\Gamma_{k j}^{\sigma} \Gamma_{l i}^{h}-\Gamma_{k i}^{\sigma} \Gamma_{l j}^{h}\right] .
\end{aligned}
$$

Evidently, in view of equations (1.29), (1.33), (1.34), (1.35), (1.36), (1.37) and (1.38), the Weyl's tensor density can be evolved as the composition of components of Riemannian tensor density $R_{i j k l}^{*}$, the Ricci tensor densities $R_{j l}^{*}, R_{j k}^{*}, R_{i k}^{*}, R *_{i l}$ and the scalar curvature density $R^{*}$ in the following way:

$$
W_{i j k l}^{*}=R_{i j k l}^{*}-\frac{\left(R_{i k}^{*} g_{j l}-R_{i l}^{*} g_{j k}+R_{j l}^{*} g_{i k}-R_{j k}^{*} g_{i l}\right)}{2}+\frac{R^{*}\left(g_{i k} g_{j l}-g_{i l} g_{j k}\right)}{6} .
$$




\subsubsection{Evolution of Weylian Configurations under Ricci Flow:}

It has been already mentioned in the foregoing section that in addition to completely analyze the geometric nature of structured cosmos of dimension $n=4$, (from axiomatic as weel as observational stand points), one need to go through conformally Riemannian structures (often called Weylian configurations) rather than Riemannian one.

To develop the structured cosmos, Weyl's affine connection given by eq. (1.26) has been introduced. Also, a Weyl's tensor density given by eq. (1.39) has been setup. It is also known that Weylian configurations given by equations (1.26), (1.32), (1.33), (1.34), (1.35), (1.36), (1.37), (1.38) and (1.39) are more rigid than topological structures but, more flexible than Riemannian structures. Therefore, we now evolve the heat diffusion equations (i.e., Ricci Flow) of Weylian configurations by making use of the following basic notions:

The Ricci flow (in abbreviated form "R.F.") is a mean by which one can take an arbitrary Riemannian manifold and smooth out geometry of that manifold to make it look more symmetric. Informally, R.F. is the process of stretching the metric tensor $g_{i j}$ in the direction of negative Ricci curvature and contracting the same in the direction of positive Ricci curvature. Thus, concisely and lucidly, the R.F. can be delineated by the equation [3]:

$$
\frac{d}{d t} g=-2 \mathrm{Ric} \equiv \partial_{t} g_{i j}=-2 R_{i j}
$$

Now under the R.F. (1.40), the metric tensor given by eq. (1.28) turns out to be a no-linear heat diffusion equation for Riemannian curvature tensor (1.27), as follows:

$$
\begin{aligned}
& \frac{\partial}{\partial t} R_{i j k l}=\nabla_{g}^{2} R_{i j k l}+2\left(D_{i j k l}-D_{i j l k}-D_{i l j k}+D_{i k j l}\right) \\
& -g^{p q}\left(R_{p j k l} R_{q i}+R_{i p k l} R_{q j}+R_{i j p l} R_{q k}+R_{i j k p} R_{q l}\right)
\end{aligned}
$$

where $D_{i j k l}=g^{p r} g^{q s} R_{p i q j} R_{r k s l}$ and $\nabla_{g}^{2}$ is the Laplacian with respect to the evolving metric (1.28) and is defined as;

$$
\nabla_{g}^{2}=g^{i j} \nabla_{i} \nabla_{j}=g^{i j}\left(\frac{\partial^{2}}{\partial x^{i} \partial x^{j}}-\Gamma_{i j}^{k} \frac{\partial}{\partial x^{k}}\right) .
$$

Further, the heat diffusion equation for Ricci curvature tensor $R_{i k}$ is given by;

$$
\frac{\partial}{\partial t} R_{i k}=\nabla_{g}^{2} R_{i k}+2 g^{p r} g^{q s} R_{p i q k} R_{r s}-2 g^{p q} R_{p i} R_{q k}
$$

while the heat diffusion equation for scalar curvature $R$ is

$$
\frac{\partial R}{\partial t}=\nabla_{g}^{2} R+2 \mathrm{Ric}^{2}
$$


where $\operatorname{Ric}^{2}=R_{p q} R_{i k} g^{i p} g^{q k}$ is called length of $R_{i k}$.

Moreover, the Ricci flow of Christoffel's symbol $\Gamma_{i j}^{k}$ is of the form:

$$
\frac{\partial}{\partial t} \Gamma_{i j}^{k}=-\frac{1}{2} g^{k l}\left(R_{j l ; i}+R_{i l ; j}-R_{i j ; l}\right)=\nabla \mathrm{Rm} .
$$

In case of conformal Riemannian (Weylian) geometry, of course, the curvatures are not only the significant Weylian configurations. But, the volume structures of the manifold are also of fundamental importance and interest. The evolution of the volume element $d v=\sqrt{\left(\operatorname{det} g_{i j}\right)} d x$ of the space-time $M$ is given by:

$$
\frac{\partial}{\partial t} \log \sqrt{\left(\operatorname{det} g_{i j}\right)} d x=\frac{1}{2} g^{i j} \frac{\partial}{\partial t} g_{i j}=r-R,
$$

where $r=\frac{\int_{M} R d v}{\int_{M} d v}$ is the average scalar curvature and serves to normalize the R.F., so that the volume is constant. But, most often, the volume is not constant for the structured cosmos due to different layers of atmosphere, therefore, to prevent the solution of eq. (1.45) from shrinking to a point or expanding to $\infty$, we consider the normalized R.F.:

$$
\frac{\partial}{\partial t} g_{i j}=\frac{2}{n} r g_{i j}-2 R_{i j},
$$

so that

$$
\frac{\partial}{\partial t} \log \sqrt{\left(\operatorname{det} g_{i j}\right)}=0 \text {. }
$$

Here, in our case the space-time is of dimension $n=4$.

Now, with the help of all the expressions mentioned in this sub-subsection, we evolve the following Weyl's configurations under R.F.:

\subsubsection{Evolution of Heat Diffusion Equation for $\Upsilon_{j k}^{i}$ under R.F.}

Let us evolve the heat diffusion equation for Weyl's affine connection eq. (1.26), by taking care of equation (1.40), (1.41), (1.42), (1.43), (1.44), (1.45), (1.46) and (1.47) as below:

Taking R.F. of Weyl's affine connection given by eq. (1.26), we get

$$
\begin{gathered}
\frac{\partial}{\partial t} \Upsilon_{j k}^{i}=\frac{1}{2} \frac{\partial}{\partial t} g^{i h}\left(g_{j h, k}+g_{k h, j}-g_{j k, h}\right)+\frac{1}{4} \frac{\partial}{\partial t} g^{i h}\left(g_{j h} \phi_{k}+g_{k h} \phi_{j}-g_{j k} \phi_{h}\right) \\
-\frac{1}{2} \frac{\partial}{\partial t}\left(\phi_{k} \delta_{j}^{i}+\phi_{j} \delta_{k}^{i}-g_{j k} \phi^{i}\right)
\end{gathered}
$$




$$
\begin{aligned}
& =\frac{1}{2}\left[g^{i h} \frac{\partial}{\partial t}\left(g_{j h, k}+g_{k h, j}-g_{j k, h}\right)+\left(g_{j h, k}+g_{k h, j}-g_{j k, h} \frac{\partial}{\partial t} g^{i h}\right)\right] \\
& +\frac{1}{4}\left[g^{i h} \frac{\partial}{\partial t}\left(g_{j h} \phi_{k}+g_{k h} \phi_{j}-g_{j k} \phi_{h}\right)+\left(g_{j h} \phi_{k}+g_{k h} \phi_{j}-g_{j k} \phi_{h}\right) \frac{\partial}{\partial t} g^{i h}\right] \\
& -\frac{1}{2}\left[\phi_{k} \frac{\partial}{\partial t} \delta_{j}^{i}+\delta_{j}^{i} \frac{\partial \phi_{k}}{\partial t}+\phi_{j} \frac{\partial}{\partial t} \delta_{k}^{i}+\delta_{k}^{i} \frac{\partial \phi_{j}}{\partial t}-g_{j k} \frac{\partial \phi^{i}}{\partial t}-\phi^{i} \frac{\partial g_{j k}}{\partial t}\right] . \\
& =\frac{1}{2}\left[g^{i h}\left(\frac{\partial}{\partial x^{k}} \frac{\partial}{\partial t} g_{j h}+\frac{\partial}{\partial x^{j}} \frac{\partial}{\partial t} g_{k h}-\frac{\partial}{\partial x^{h}} \frac{\partial}{\partial t} g_{j k}\right)+\left(\frac{\partial}{\partial x^{k}} g_{j h}+\frac{\partial}{\partial x^{j}} g_{k h}-\frac{\partial}{\partial x^{h}} g_{j k}\right) g^{i p}\right. \\
& \left.\left(\frac{\partial}{\partial t} g_{p q}\right)\right]+\frac{1}{4}\left[g^{i h}\left(\phi_{k} \frac{\partial g_{j h}}{\partial t}+g_{j h} \frac{\partial \phi_{k}}{\partial t}+\phi_{j} \frac{\partial g_{k h}}{\partial t}+g_{k h} \frac{\partial \phi_{j}}{\partial t}-\phi_{h} \frac{\partial g_{j k}}{\partial t}-g_{j k} \frac{\partial \phi_{h}}{\partial t}\right)\right. \\
& \left.+\left(g_{j h} \phi_{k}+g_{k h} \phi_{j}-g_{j k} \phi_{h}\right) g^{i p}\left(\frac{\partial}{\partial t} g_{p q}\right) g^{h q}\right]-\frac{1}{2}\left[\phi_{k} \frac{\partial}{\partial t}\left(g^{i p} g_{j p}\right)+g^{i p} g_{j p} \frac{\partial \phi_{k}}{\partial t}+\phi_{j} \frac{\partial}{\partial t}\left(g^{i p} g_{k p}\right)\right. \\
& \left.+g^{i p} g_{k p} \frac{\partial \phi_{j}}{\partial t}-\phi^{i} \frac{\partial g_{j k}}{\partial t}-g_{j k} \frac{\partial \phi^{i}}{\partial t}\right] . \\
& =\frac{1}{2}\left[g^{i h}\left(\frac{\partial}{\partial x^{k}} \frac{\partial}{\partial t} g_{j h}+\frac{\partial}{\partial x^{j}} \frac{\partial}{\partial t} g_{k h}-\frac{\partial}{\partial x^{h}} \frac{\partial}{\partial t} g_{j k}\right)+\left(\frac{\partial}{\partial x^{k}} h_{j h}+\frac{\partial}{\partial x^{j}} g_{k h}-\frac{\partial}{\partial x^{h}} g_{j k}\right) \times\right. \\
& \left.g^{i p}\left(\frac{\partial}{\partial t} g_{p q}\right) g^{h q}\right]+\frac{1}{4}\left[g^{i h}\left(\phi_{k} \frac{\partial g_{j h}}{\partial t}+g_{j h} \frac{\partial \phi_{k}}{\partial t}+\phi_{j} \frac{\partial g_{k h}}{\partial t}+g_{k h} \frac{\partial \phi_{j}}{\partial t}-\phi_{h} \frac{\partial g_{j k}}{\partial t}-g_{j k} \frac{\partial \phi_{h}}{\partial t}\right)\right. \\
& \left.+\left(g_{j h} \phi_{k}+g_{k h} \phi_{j}-g_{j k} \phi_{h}\right) g^{i p}\left(\frac{\partial}{\partial t} g_{p q}\right) g^{h q}\right]-\frac{1}{2}\left[\phi_{k} g^{i p} \frac{\partial g_{j p}}{\partial t}+\phi_{k} g_{j p} g^{i m}\left(\frac{\partial}{\partial t} g_{m n}\right) g^{p n}\right. \\
& \left.+g^{i p} g_{j p} \frac{\partial \phi_{k}}{\partial t}+\phi_{j} g^{i p} \frac{\partial g_{k p}}{\partial t}+\phi_{j} g_{k p} g^{i m}\left(\frac{\partial}{\partial t} g_{m n}\right) g^{p m}+g^{i p} g_{k p} \frac{\partial \phi_{j}}{\partial t}-g_{j k} \frac{\partial \phi^{i}}{\partial t}-\phi^{i} \frac{\partial \phi^{i}}{\partial t}\right] .
\end{aligned}
$$

Here, in the above manipulation, we have used the following basic tensorial algebra:

(i) $\frac{\partial}{\partial t} g^{i h}=g^{i p}\left(\frac{\partial}{\partial t} g_{p q}\right) g^{h q}$ etc..(ii) $g_{j h, k} \equiv \frac{\partial}{\partial x^{k}} g_{j h}$ etc..(iii) $\delta_{j}^{i}=g^{i p} g_{j p}$. 
Now, under the R.F. techniques, the last lengthy expression reduces to:

$$
\begin{gathered}
\frac{\partial}{\partial t} \Upsilon_{j k}^{i}=\left[g^{i h}\left(\frac{\partial}{\partial x^{h}} R_{j k}-\frac{\partial}{\partial x^{j}} R_{k h}-\frac{\partial}{\partial x^{k}} R_{j h}-2\{j k, h\} g^{i p} g^{h q} R_{p q}\right)\right] \\
+\frac{1}{4}\left[g^{i h}\left(-2 \phi_{k} R_{j h}+g_{j h} \frac{\partial \phi_{k}}{\partial t}-2 \phi_{j} R_{k h}+g_{k h} \frac{\partial \phi_{j}}{\partial t}+2 \phi_{h} R_{j k}-g_{j k} \frac{\partial \phi_{h}}{\partial t}\right)\right. \\
\left.-2\left(g_{j h} \phi_{k}+g_{k h} \phi_{j}-g_{j k} \phi_{h}\right) g^{i p} g^{h q} R_{p q}\right]-\frac{1}{2}\left[-2 \phi_{k} g^{i p} R_{j p}-2 \phi_{k} g_{j p} g^{i m} g^{p m} R_{m n}\right. \\
\left.+g^{i p} g_{j p} \frac{\partial \phi_{k}}{\partial t}-2 \phi_{j} g^{i p} R_{k p}-2 \phi_{j} g_{k p} g^{i p} g^{p m} R_{m n}+g^{i p} g_{k p} \frac{\partial \phi_{j}}{\partial t}-g_{j k} \frac{\partial \phi^{i}}{\partial t}+2 \phi^{i} R_{j k}\right]
\end{gathered}
$$

\subsubsection{Evolution of Heat Diffusion Equation for $R_{\mathrm{ijkl}}^{*}$ under R.F.}

We evolve the Riemannian tensor density $R_{i j k l}^{*}$ eq. (1.33) under R. F. in the following fashion:

Taking R.F. on both side of eq. (1.33), we get

$$
\begin{gathered}
R_{i j k l}^{*}=\frac{\partial}{\partial x^{j}}\left[g_{h l} \frac{\partial}{\partial t} \Gamma_{k i}^{h}+\Gamma_{k i}^{h} \frac{\partial}{\partial t} g_{h l}\right]+\frac{1}{2}\left[\phi_{j} g_{h l} \frac{\partial}{\partial t} \Gamma_{k i}^{h}+\phi_{j} \Gamma_{k i}^{h} \frac{\partial}{\partial t} g_{h l}+g_{h l} \Gamma_{k i}^{h} \frac{\partial \phi_{j}}{\partial t}\right] \times \\
-\frac{\partial}{\partial x^{i}}\left[g_{h l} \frac{\partial}{\partial t} \Gamma_{k j}^{h}+\Gamma_{k j}^{h} \frac{\partial}{\partial t} g_{h l}\right]-\frac{1}{2}\left[\phi_{j} g_{h l} \frac{\partial}{\partial t} \Gamma_{k j}^{h}+\phi_{i} \Gamma_{k j}^{h} \frac{\partial}{\partial t} g_{h l}+g_{h l} \Gamma_{k j}^{h} \frac{\partial \phi_{i}}{\partial t}\right] \\
+g_{h \sigma} \Gamma_{l i}^{h} \frac{\partial}{\partial t} \Gamma_{k j}^{\sigma}-g_{h \sigma} \Gamma_{k i}^{\sigma} \frac{\partial}{\partial t} \Gamma_{l j}^{h}-g_{h \sigma} \Gamma_{l j}^{h} \frac{\partial}{\partial t} \Gamma_{k i}^{\sigma}+\left[\Gamma_{k j}^{\sigma} \Gamma_{l i}^{h}-\Gamma_{k i}^{\sigma} \Gamma_{l j}^{h}\right] \frac{\partial g_{h \sigma}}{\partial t} .
\end{gathered}
$$

Now under R.F. techniques, the last expression reduces to:

$$
\begin{gathered}
\frac{\partial}{\partial t} R_{i j k l}^{*}=-\frac{\partial}{\partial x^{j}}\left[g_{h l} g^{h l} \frac{1}{2}\left(R_{i l ; k}+R_{k l ; i}-R_{k i ; l}\right)+2 \Gamma_{k i}^{h} R_{h l}\right] \\
-\frac{1}{2}\left[\phi_{j} g_{h l} g^{h l} \frac{1}{2}\left(R_{i l ; k}+R_{k l ; i}-R_{k i ; l}\right)+2 \phi_{j} \Gamma_{k i}^{h} R_{h l}-g_{h l} \Gamma_{k i}^{h} \frac{\partial \phi_{j}}{\partial t}\right]
\end{gathered}
$$




$$
\begin{gathered}
+\frac{\partial}{\partial x^{i}}\left[g_{h l} g^{h l} \frac{1}{2}\left(R_{j l ; k}+R_{k l ; j}-R_{k j ; l}\right)+2 \Gamma_{k j}^{h} R_{h l}\right]+\frac{1}{2}\left[\phi_{i} g_{h l} g^{h l} \frac{1}{2}\left(R_{j l ; k}+R_{k l ; j}-R_{k j ; l}\right)+\right. \\
\left.+2 \phi_{i} \Gamma_{k j}^{h} R_{h l}-g_{h l} \Gamma_{k j}^{h} \frac{\partial \phi_{i}}{\partial t}\right]-\frac{1}{2}\left[g_{h \sigma} \Gamma_{k j}^{\sigma} g^{h w}\left(R_{i w ; l}+R_{l w ; i}-R_{i l ; w}\right)\right. \\
+g_{h \sigma} \Gamma_{l i}^{h} g^{\sigma w}\left(R_{k w ; j}+R_{j w ; k}-R_{k j ; w}\right)-g_{h \sigma} \Gamma_{k i}^{\sigma} g^{g w}\left(R_{j w ; l}+R_{l w ; j}-R_{l j ; w}\right) \\
\left.-g_{h \sigma} \Gamma_{l j}^{h} g^{\sigma w}\left(R_{k w ; i}+R_{i w ; k}-R_{k i ; w}\right)\right]-2 R_{h \sigma}\left(\Gamma_{k j}^{\sigma} \Gamma_{l i}^{h}-\Gamma_{k i}^{\sigma} \Gamma_{l j}^{h} .\right)
\end{gathered}
$$

\subsubsection{Evolution of Heat Diffusion Equations for the Components of Ricci tensor density under R.F.}

Let us now evolve the components of Ricci tensor density given by equations (1.34), (1.35), (1.36) and (1.37) in the following way:

Taking the R.F. on both side of eq. (1.34) and manipulating under the conditions $(i),(i i)$ and $(i i i)$, we obtain:

$$
\begin{gathered}
\frac{\partial}{\partial t} R_{j l}^{*}=g^{i k}\left[\frac{\partial}{\partial x^{j}}\left(g_{h l} \frac{\partial}{\partial t} \Gamma_{k i}^{h}+\Gamma_{k i}^{h} \frac{\partial}{\partial t} g_{h l}\right)\right]+\frac{\partial}{\partial x^{j}}\left(g_{h l} \Gamma_{k i}^{h}\right) g^{i m}\left(\frac{\partial}{\partial t} g_{m n}\right) g^{k n} \\
+\frac{1}{2}\left[\phi_{j}\left(g^{i k} g_{h l} \frac{\partial}{\partial t} \Gamma_{k i}^{h}+g^{i k} \Gamma_{k i}^{h} \frac{\partial}{\partial t} g_{h l}+g_{h l} \Gamma_{k i}^{h} g^{i m}\left\{\frac{\partial}{\partial t} g_{m n}\right\} g^{k n}\right)+g^{i k} g_{h l} \Gamma_{k i}^{h} \frac{\partial}{\partial t} \phi_{j}\right] \\
-g^{i k}\left[\frac{\partial}{\partial x^{i}}\left(g_{h l} \frac{\partial}{\partial t} \Gamma_{k j}^{h}+\Gamma_{k j}^{h} \frac{\partial}{\partial t} g_{h l}\right)\right]-\frac{\partial}{\partial x^{i}}\left(g_{h l} \Gamma_{k j}^{h}\right) g^{i m}\left(\frac{\partial}{\partial t} g_{m n}\right) g^{k n} \\
-\frac{1}{2}\left[\phi_{i}\left(g^{i} k g_{h l} \frac{\partial}{\partial t} \Gamma_{k j}^{h}+g^{i k} \Gamma_{k j}^{h} \frac{\partial}{\partial t} g_{h l}+g_{h l} \Gamma_{k j}^{h} g^{i m}\left(\frac{\partial}{\partial t} g_{m n}\right) g^{k n}\right)+g^{i k} g_{h l} \Gamma_{k j}^{h} \frac{\partial}{\partial t} \phi_{i}\right] \\
+g^{i k}\left[g_{h \sigma}\left(\Gamma_{k j}^{\sigma} \frac{\partial}{\partial t} \Gamma_{l i}^{h}+\Gamma_{l i}^{h} \frac{\partial}{\partial t} \Gamma_{k j}^{\sigma}-\Gamma_{k i}^{\sigma} \frac{\partial}{\partial t} \Gamma_{l j}^{h}-\Gamma_{l j}^{h} \frac{\partial}{\partial t} \Gamma_{k i}^{\sigma}\right)+\left(\Gamma_{k j}^{\sigma} \Gamma_{l i}^{h}-\Gamma_{k i}^{\sigma} \Gamma_{l j}^{h}\right) \frac{\partial}{\partial t} g_{h \sigma}\right] \\
+g_{h \sigma}\left(\Gamma_{k j}^{\sigma} \Gamma_{l i}^{h}-\Gamma_{k i}^{\sigma} \Gamma_{l j}^{h}\right) \frac{\partial}{\partial t} g^{i k} .
\end{gathered}
$$


Under the R.F. techniques, the last expression eventually produces;

$$
\begin{gathered}
\frac{\partial}{\partial t} R_{j l}^{*}=g^{i k}\left[\frac{\partial}{\partial x^{j}}\left(-\frac{1}{2} g_{h l} g^{h l}\left(R_{i l: k}+R_{k l ; i}-R_{k i ; l}\right)-2 R_{h l} \Gamma_{k i}^{h}\right)\right] \\
-2 \frac{\partial}{\partial x^{j}}\left(g_{h l} \Gamma_{k i}^{h}\right) g^{i m} g^{k n} R_{m n}++\frac{1}{2}\left[\phi _ { j } \left(-\frac{1}{2} g^{i k} g^{h l} g_{h l}\left(R_{i k ; l}+R_{k l ; i}-R_{k i ; l}\right)\right.\right. \\
\left.\left.-2 R_{h l} g^{i k} \Gamma_{k i}^{h}-2 g_{h l} \Gamma_{k i}^{h} g^{i m} g^{k n} R_{m n}\right)+g^{i k} g_{h l} \Gamma_{k j}^{h} \frac{\partial \phi_{j}}{\partial t}\right]+g^{i k}\left[\frac { \partial } { \partial x ^ { i } } \left\{\frac{1}{2} g_{h l} g^{h l}\left(R_{j l ; k}+R_{k l ; j}-R_{k j ; l}\right)\right.\right. \\
\left.\left.+2 R_{h l} \Gamma_{k j}^{h}\right\}\right]+2 \frac{\partial}{\partial x^{i}}\left(g_{h l} \Gamma_{k j}^{h}\right) g^{i m} g^{k n} R_{m n}+\frac{1}{2}\left[\phi _ { i } \left\{\frac{1}{2} g^{i k} g_{h l} g^{h l}\left(R_{j l ; k}+R_{k l ; j}-R_{k j ; l}\right)\right.\right. \\
\left.\left.+2 R_{h l} g^{i k} \Gamma_{k j}^{h}+2 g_{h l} \Gamma_{k j}^{h} g^{i m} g^{k n} R_{m n}\right\}-g^{i k} g_{h l} \Gamma_{k j}^{h} \frac{\partial \phi_{i}}{\partial t}\right]-g^{i k}\left[g _ { h \sigma } \left\{\frac{1}{2} \Gamma_{k j}^{\sigma} g^{h u}\left(R_{l u ; i}+R_{i u ; l}-R_{l i ; u}\right)\right.\right. \\
+\frac{1}{2} \Gamma_{l i}^{h} g^{\sigma u}\left(R_{k u ; j}+R_{j u ; k}-R_{k j ; u}\right)-\frac{1}{2} \Gamma_{k i}^{\sigma} g^{h u}\left(R_{l u ; j}+R_{j u ; l}-R_{l j ; u}\right) \\
\left.\left.-\frac{1}{2} \Gamma_{l j}^{h} g^{\sigma u}\left(R_{k u ; i}+R_{i u ; k}-R_{k i ; u}\right)\right\}+2 R_{h \sigma}\left(\Gamma_{k j}^{\sigma} \Gamma_{l i}^{h}-\Gamma_{k i}^{\sigma} \Gamma_{l j}^{h}\right)\right]-2 g_{h \sigma} g^{i m} g^{l n} R_{m n}\left(\Gamma_{k j}^{\sigma} \Gamma_{l i}^{h}-\Gamma_{k i}^{\sigma} \Gamma_{l j}^{h}\right) .
\end{gathered}
$$

Further, taking R.F. on both side of eq. (1.35) performing some lengthy but straight forward in the same way as above, we obtain

$$
\begin{gathered}
\frac{\partial}{\partial t} R_{j k}^{*}=-g^{i l}\left[\frac{\partial}{\partial x^{j}}\left\{\frac{1}{2} g_{h l} g^{h l}\left(R_{i l ; k}+R_{k l ; j}-R_{k i ; l}\right)+2 R_{h l} \Gamma_{k i}^{h}\right\}\right]-2 g^{i m} g^{l n} R_{m n} \frac{\partial}{\partial t}\left(g_{h l} \Gamma_{k i}^{h}\right) \\
+\frac{1}{4} \frac{\partial \log \left(g_{i i}\right)}{\partial x^{k}} \frac{\partial \phi_{j}}{\partial t}+g^{i l}\left[\frac{\partial}{\partial x^{i}}\left\{\frac{1}{2} g_{h l} g^{h l}\left(R_{k l ; j}+R_{j l ; k}-R_{k j ; l}\right)+2 R_{h l} \Gamma_{k j}^{h}\right\}\right] \\
+2 g^{i m} g^{l n} R_{m n} \frac{\partial}{\partial x^{i}}\left(g_{h l} \Gamma_{k j}^{h}\right)+\frac{1}{2}\left\{\frac{1}{2} \phi_{i} g_{i l}\left(R_{k l ; i}+R_{j l ; k}-R_{k j ; l}\right)-\Gamma_{k j}^{i} \frac{\partial \phi_{i}}{\partial t}\right\} \\
-g^{i l}\left[g _ { h \sigma } \left\{\frac{1}{2} \Gamma_{k j}^{\sigma} g^{h u}\left(R_{l u ; i}+R_{i u ; l}-R_{l i ; u}\right)+\frac{1}{2} \Gamma_{l i}^{h} g^{\sigma u}\left(R_{k u ; j}+R_{j u ; k}-R_{k j ; u}\right)\right.\right.
\end{gathered}
$$




$$
\begin{gathered}
\left.-\frac{1}{2} \Gamma_{k i}^{\sigma} g^{h u}\left(R_{l u ; j}+R_{j u ; l}-R_{l j ; u}\right)-\frac{1}{2} \Gamma_{l j}^{h} g^{\sigma u}\left(R_{k u ; i}+R_{i u ; k}-R_{k i ; u}\right)\right\} \\
\left.+2 R_{h \sigma}\left(\Gamma_{k j}^{\sigma} \Gamma_{l i}^{h}-\Gamma_{k i}^{\sigma} \Gamma_{l j}^{h}\right)\right]-2 g_{h \sigma} g^{i m} g^{l n} R_{m n}\left(\Gamma_{k j}^{\sigma} \Gamma_{l i}^{h}-\Gamma_{k i}^{\sigma} \Gamma_{l j}^{h}\right) .
\end{gathered}
$$

Afterward, taking R.F. on both side of the eq. (1.36) and going through complicated manipulations as above, we arrive at the final result as:

$$
\begin{aligned}
& \frac{\partial}{\partial t} R^{*} i l=-g^{j k}\left[\frac{\partial}{\partial x^{j}}\left\{\frac{1}{2} g_{h l} g^{h l}\left(R_{k l ; i}+R_{i l ; k}-R_{k i ; l}\right)+2 R_{h l} \Gamma_{k i}^{h}\right\}\right] \\
& -2 \frac{\partial}{\partial x^{j}}\left(g_{h l} \Gamma_{k i}^{h}\right) g^{j m} g^{k n} R_{m n}+\frac{1}{2}\left[\phi _ { j } \left\{-\frac{1}{2} g^{j k} g_{h l} g^{h l}\left(R_{k l ; i}+R_{i l ; k}-R_{k i ; l}\right)\right.\right. \\
& \left.\left.-2 g^{j k} \Gamma_{k i}^{h} R_{h l}-2\left(g_{h l} \Gamma_{k i}^{h}\right) g^{j m} g^{k n} R_{m n}\right\}+g^{j k} g_{h l} \Gamma_{k i}^{h} \frac{\partial \phi_{j}}{\partial t}\right]-g^{j k}\left\{\frac { \partial } { \partial x ^ { i } } \left\{-\frac{1}{2} g_{h l} g^{h l} \times\right.\right. \\
& \left.\left.\left(R_{k l ; j}+R_{j l ; k}-R_{k j ; l}\right)-2 g^{j k} \Gamma_{k i}^{h} R_{h l}-2 g_{h l} \Gamma_{k i}^{h} g^{j} m g^{k n} R_{m n}\right\}+g^{j k} g_{h l} \Gamma_{k i}^{h} \frac{\partial \phi_{j}}{\partial t}\right] \\
& -\frac{1}{2}\left[\phi_{i}\left\{-\frac{1}{2} g^{j k} g_{h l} g^{h l}\left(R_{k l ; j}+R_{j l ; k}-R_{k j ; l}\right)+-2 \Gamma_{k i}^{h} g^{j k} R_{h l}-2 \Gamma_{k j}^{h} g_{h l} g^{m j} g^{k n} R_{m n}\right\}\right. \\
& \left.+g^{j k} g_{h l} \Gamma_{k j}^{h} \frac{\partial \phi_{i}}{\partial t}\right]-g^{j k}\left[g _ { h \sigma } \left\{\frac{1}{2} \Gamma_{k j}^{\sigma} g^{h u}\left(R_{l u ; i}+R_{i u ; l}-R_{l i ; u}\right)+\frac{1}{2} \Gamma_{l i}^{h} g^{\sigma u}\left(R_{k u ; j}+R_{j u ; k}-R_{k j ; u}\right)\right.\right. \\
& \left.-\frac{1}{2} \Gamma_{k i}^{\sigma} g^{h u}\left(R_{l u ; j}+R_{j u ; l}-R_{l j ; u}\right)-\frac{1}{2} \Gamma_{l j}^{h} g^{\sigma u}\left(R_{k u ; i}+R_{i u ; k}-R_{k i ; u}\right)\right\}+2 R_{h \sigma}\left(\Gamma_{k j}^{\sigma} \Gamma_{l i}^{h}\right. \\
& \left.\left.-\Gamma_{k i}^{\sigma} \Gamma_{l j}^{h}\right)\right]-2 g_{h \sigma} g^{j m} g^{k n} R_{m n}\left(\Gamma_{k j}^{\sigma} \Gamma_{l i}^{h}-\Gamma_{k i}^{\sigma} \Gamma_{l j}^{h}\right) .(1.52) \\
& \text { Moreover, taking R.F. on both side of eq.(1.37) and solving for obtaining some } \\
& \text { feasible form, we get } \\
& \begin{array}{c}
\frac{\partial}{\partial t} R_{i k}^{*}=-g^{j l}\left[\frac{\partial}{\partial x^{j}}\left\{\frac{1}{2} g_{h l} g^{h l}\left(R_{k l ; i}+R_{i l ; k}-R_{k i ; l}\right)\right\}+2 R_{h l} \Gamma_{k i}^{h}\right]-2 g^{j m} g^{l n} R_{m n} \frac{\partial}{\partial x^{j}}\left(g_{h l} \Gamma_{k i}^{h}\right) \\
\frac{1}{4} \phi_{j} \frac{\partial \log \left(g_{j j}\right)}{\partial x^{k}} \frac{\partial \phi_{j}}{\partial t}+g^{j l}\left[\frac{\partial}{\partial x^{i}}\left\{\frac{1}{2} g_{h l} g^{h l}\left(R_{k l ; j}+R_{j l ; k}-R_{k j ; l}+2 R_{h l} \Gamma_{k j}^{h}\right)\right\}\right]
\end{array}
\end{aligned}
$$




$$
\begin{gathered}
+2 \frac{\partial}{\partial x^{i}}\left(g_{h l} \Gamma_{k j}^{h}\right) g^{j m} g^{l n} R_{m n}+\frac{1}{2}\left[\Gamma_{k i}^{j} \frac{\partial \phi_{j}}{\partial t}-\frac{1}{2} \phi_{j} g^{j l}\left(R_{k l ; i}+R_{i l ; k}-R_{k i ; l}\right)\right] \\
-g^{j l}\left[g _ { h \sigma } \left\{\frac{1}{2} \Gamma_{k j}^{\sigma} g^{h u}\left(R_{l u ; i}+R_{i u ; l}-R_{l i ; u}\right)+\frac{1}{2} \Gamma_{l i}^{h} g^{\sigma u}\left(R_{k u ; j}+R_{j u ; k}-R_{k j ; u}\right)-\frac{1}{2} \times\right.\right. \\
\left.\left.\Gamma_{k i}^{\sigma} g^{h u}\left(R_{l u ; j}+R_{j u ; l}-R_{l j ; u}\right)-\frac{1}{2} \Gamma_{j l}^{h} g^{\sigma u}\left(R_{k u ; i}+R_{i u ; k}-R_{k i ; u}\right)\right\}+2 R_{h \sigma}\left(\Gamma_{k j}^{\sigma} \Gamma_{l i}^{h}-\Gamma_{k i}^{\sigma} \Gamma_{l j}^{h}\right)\right] \\
-2 g_{h \sigma} g^{j m} g^{k n} R_{m n}\left(\Gamma_{k j}^{\sigma} \Gamma_{l i}^{h}-\Gamma_{k i}^{\sigma} \Gamma_{l j}^{h}\right) .
\end{gathered}
$$

Eventually, we now take R.F. of eq. (1.38) and simplify with the help of known R.F. techniques, we obtain the resulting heat flow equation as:

$$
\begin{gathered}
\frac{\partial}{\partial t} R^{*}=-g^{i j}\left[g^{k k l} \frac{\partial}{\partial x^{j}}\left\{\frac{1}{2} g_{h l} g^{h l}\left(R_{k l ; i}+R_{i l ; k}-R_{k i, l}\right)+2 R_{h l} \Gamma_{k i}^{h}\right\}\right. \\
\left.+2 g^{k m} g^{l n} R_{m n} \frac{\partial}{\partial x^{j}}\left(g_{h l} \Gamma_{k i}^{h}\right)\right]-2 g^{i m} g^{j n} R_{m n} g^{k l} \frac{\partial}{\partial x^{i}}\left(g_{h l} \Gamma_{k i}^{h}\right)+\frac{1}{4}\left[\frac{\partial}{\partial x^{i}} \log \left(g_{k k}\right) \frac{\partial \phi^{i}}{\partial t}\right] \\
+g^{i j}\left[g^{k l} \frac{\partial}{\partial x^{i}}\left\{\frac{1}{2} g_{h l} g^{h l}\left(R_{k l ; j}+R_{j l ; k}-R_{k j ; l}\right)+2 R_{h l} \Gamma_{k j}^{h}\right\}+2 g^{k m} g^{l n} R_{m n} \frac{\partial}{\partial x^{i}}\left(g_{h l} \Gamma_{k j}^{h}\right)\right] \\
-2 R_{m n} g^{i m} g^{j n} g^{k l} \frac{\partial}{\partial x^{i}}\left(g_{h l} \Gamma_{k j}^{h}\right)-\frac{1}{4}\left[\frac{\partial}{\partial x^{i}} \log \left(g_{k k}\right) \frac{\partial \phi^{i}}{\partial t}\right]-g^{i j}\left[g ^ { k l } \left\{g _ { g \sigma } \left(\frac{1}{2} \Gamma_{k j}^{\sigma} g^{h u} \times\right.\right.\right. \\
\left(R_{l u ; i}+R_{i u ; l}-R_{l i ; u}\right)+\frac{1}{2} \Gamma_{l i}^{h} g^{\sigma u}\left(R_{k u ; j}+R_{j u ; k}-R_{j k ; u}\right)-\frac{1}{2} \Gamma^{\sigma} g^{h u}\left(R_{l u ; j}+R_{j u ; l}-R_{l j ; u}\right) \\
\left.\left.-\frac{1}{2} \Gamma_{l j}^{h} g^{\sigma u}\left(R_{k u ; i}+R_{i u ; k}-R_{k i ; u}\right)\right)+2 R_{h \sigma}\left(\Gamma_{k j}^{\sigma} \Gamma_{l i}^{h}-\Gamma_{k i}^{\sigma} \Gamma_{l j}^{h}\right)\right\}+2 g^{k m} g^{l n} R_{m n} g_{h \sigma} \times \\
\left.\left(\Gamma_{k j}^{\sigma} \Gamma_{l i}^{h}-\Gamma_{l i}^{\sigma} \Gamma_{k j}^{h}\right)\right]+2 R_{m n} g^{i m} g^{j n} g^{k l} g_{h \sigma}\left(\Gamma_{k j}^{\sigma} \Gamma_{l i}^{h}-\Gamma_{k i}^{\sigma} \Gamma_{l j}^{h}\right) .
\end{gathered}
$$




\subsubsection{Evolution of Heat Diffusion Equation for the Weyl's tensor density $W_{\mathrm{ijkl}}^{*}$ under R.F.}

Now, we have been arriving at the extrimity, where we can evolve the R.F. for the most desirable Weyl's tensor density in Weyl-Gauge geometry.

We take the R.F. of eq. (1.39) such that the heat diffusion expression for Weyl's tensor density evolves like below:

$\frac{\partial}{\partial t} W_{i j k l}^{*}=\frac{\partial}{\partial t}\left[R_{i j k l}^{*}-\frac{\left(R_{i k}^{*} g_{j l}+R_{j l}^{*} g_{i k}-R_{i l}^{*} g_{j k}-R_{j k}^{*} g_{i l}\right)}{2}+\frac{R^{*}\left(g_{i k} g_{j l}-g_{i l} g_{j k}\right)}{6}\right]$,

which under R.F. techniques produces;

$$
\begin{gathered}
\frac{\partial}{\partial t} W_{i j k l}^{*}=\frac{\partial}{\partial t} R_{i j k l}^{*}-\left(-2 R_{j l} R^{*} i k+g_{j l} \frac{\partial}{\partial t} R_{i k}^{*}-2 R_{i k} R_{j l}^{*}+g_{i k} \frac{\partial}{\partial t} R_{j l}^{*}+2 R_{j k} R_{i l}^{*}\right. \\
\left.-g_{j k} \frac{\partial}{\partial t} R_{i l}^{*}+2 R_{i l} R_{j k}^{*}-g_{i l} \frac{\partial}{\partial t} R_{j k}^{*}\right) / 2 \\
+\left[R^{*}\left(-R_{j l} g_{i k}-2 R_{i k} g_{j l}+2 R_{j k} g_{i l}+2 R_{i l} g_{j k}\right)+\left(g_{i k} g_{j l}-g_{i l} g_{j k}\right) \frac{\partial}{\partial t} R^{*}\right] / 6 .
\end{gathered}
$$

In eq. (1.55), by substituting the R.F.'s of all calculated Weylian configurations from equations (1.49), (1.50), (1.51), (1.52), (1.53) and (1.54), we obtain a lengthy but straight forward expression for the heat diffusion equation of Weylian tensor density $W_{i j k l}^{*}$.

\subsection{Concluding Remarks}

Here is the brief discussion over some main outcomes of this article written in favor of evolution of Weyl's-Gauge invariant geometry under R.F.:

(a): Because of the prominence of Weyl's configurations due to the natural freedom of involvement of Gauge transformations in the pursuance of cosmic structures, we have developed some new Weylian structures. These weylian structures have been manufactured in such a way that they could resolve the problems of their symmetric and anti-symmetric nature in Weyl's geometry as these properties seem to be ambigious in case of Riemannian geometry.

(b): In subsection (1.2), we have calculated the components of Ricci tensor density and scalar curvature density from the given Riemannian curvature density. Also, with the help of these, a new kind of Weyl's tensor having the involvement 
of certain density (called Weyl's tensor density) and pseudo vector has been constructed.

(c): After that, in all the subsequent sub-subsections, various Weylian structures, like Weyl's affine connection, Riemannian tensor density, Ricci tensor densities etc. have been evolved under R.F. as heat diffusion equations. And finally, in the light of all these evolution equations, the heat diffusion equation for Weyl's tensor density under R.F. has been computed.

Acknowledgement: The authors extend their hearty thanks to Uttarakhand State Council for Science and Technology (UCOST) for the financial assistance via the research project entitled The study of sub-manifolds in conformal and pseudo conformal spaces. The authors are also thankful to those researchers, whose research work and master pieces have been considered during the preparation of this article.

\section{References}

[1] G. Perelman, The entropy formula for the ricci flow and its geometric applications, Tech. Rep. arXiv. org, Nov 11, (2002).

[2] G. Perelman, Ricci flow with surgery on three manifolds, Tech. Rep. arXiv. org, March 10, (2003).

[3] H-Dong Cao and XI- Pind Zhu, A complete proof of the poincare and geometrization conjectures-Application of the Hamilton-Perelman theory of the Ricci flow, Asian J. Math., Vol. 10, No. 2, pp. 165-492, (June 2006)

[4] I. Quiros, The Weyl anomaly and the nature of the background geometry, arXiv:gr-qc/0011056v1, 15 Nov 2000.

[5] I. Suhendro, A new semi-symmetric unified field theory of the classical fields of gravity and electromagnetism, Progress in physics, Vol. 4 (2007), pp. 47-62, (2007).

[6] J. Ehlers, F. A. E. Pirani and A. Schilld, General relativity in honour of J. L. Synge, Clarendon Press, U. K., (1982).

[7] P. G. Bergmann, Introduction to the theory of relativity, Prentice-hall of India pvt., (1992).

[8] R. S. Hamilton, The Harnack estimate for the ricci flow, J. Differential Geometry, Vol. 37, pp. 225-243, (1993).

[9] V. Dzhunushaliev ans H. J. Schmidt, Phys. Lett., 1, A 267, (2000). 


\section{Sandeep K. Bahuguna}

Department of Mathematics

HNB Garhwal University

Campus Badshahi Thaul,

Tehri Garhwal,

Uttarakhand,

Zip code: 249 199,

India

e-mail: drsandeepbahuguna@rediffmail.com

and

Kailash C. Petwal

Department of Mathematics

HNB Garhwal University

Campus Badshahi Thaul,

Tehri Garhwal,

Uttarakhand,

Zip code: 249 199,

India

e-mail : drkcpetwal@rediffmail.com 